Résumés des conférences et travaux

\title{
Histoire et philologie du bouddhisme chinois médiéval : sources primaires manuscrites et iconographiques
}

Costantino Moretti

\section{(2) OpenEdition}

Édition électronique

URL : https://journals.openedition.org/ashp/3972

DOI : 10.4000/ashp.3972

ISSN : 1969-6310

Éditeur

Publications de l'École Pratique des Hautes Études

\section{Édition imprimée}

Date de publication : 1 septembre 2020

Pagination : 400-401

ISSN : 0766-0677

\section{Référence électronique}

Costantino Moretti, «Histoire et philologie du bouddhisme chinois médiéval : sources primaires manuscrites et iconographiques ", Annuaire de l'École pratique des hautes études (EPHE), Section des sciences historiques et philologiques [En ligne], 151 | 2020, mis en ligne le 09 juillet 2020, consulté le 06 juillet 2021. URL : http://journals.openedition.org/ashp/3972 ; DOI : https://doi.org/10.4000/ashp. 3972 


\title{
HISTOIRE ET PHILOLOGIE DU BOUDDHISME CHINOIS MÉDIÉVAL : SOURCES PRIMAIRES MANUSCRITES ET ICONOGRAPHIQUES
}

\author{
Chargé de conférences : M. Costantino MoReTti (EFEO)
}

Programme de l'année 2018-2019: I. Lecture de textes sur l'histoire du bouddhisme chinois : le "Shi Lao zhi » (chapitre 114 du Wei shu, Livre des Wei). - II. Scènes de damnation sur les peintures murales de Dunhuang : inscriptions et éléments iconographiques.

\section{Lecture de textes sur l'histoire du bouddhisme chinois : le "Shi Lao zhi » (chapitre $114 d u$ Wei shu, Livre des Wei)}

La première conférence a été consacrée à la lecture et à l'analyse philologique du « Shi Lao zhi » 釋老志, le plus ancien traité officiel sur le bouddhisme et le taoïsme qui nous soit parvenu, un ouvrage essentiel pour étudier les évènements-clés ayant caractérisé l'histoire du bouddhisme sous les Wei du Nord (IV ${ }^{\mathrm{e}}-\mathrm{VI}^{\mathrm{e}} \mathrm{s}$. de n. è.). Ce traité apporte des renseignements détaillés sur les principaux traducteurs de textes bouddhiques de la Chine du Nord, ainsi que sur les pratiques religieuses propres à ces régions. De la même manière, il permet de mieux comprendre différents aspects liés à la gestion des monastères, à leur implantation économique, et d'analyser le rôle politique du clergé dans ce contexte historique spécifique.

Le «Shi Lao zhi », ou "Traité sur le bouddhisme et le taoïsme », constitue le chapitre 114 du Wei shu 魏書 (Livre des Wei), l'histoire officielle de la dynastie nonchinoise qui régna en Chine du Nord de 386 à 534. L'historien Wei Shou 魏收 (506572), chargé de la rédaction de cet ouvrage par l'empereur Wenxuan 文宣 (r. 550-559) des Qi du Nord (550-577), présenta officiellement ce travail au trône en 554. Toutefois, cette histoire dynastique prendra sa forma actuelle seulement une dizaine d'années plus tard, après plusieurs corrections et modifications.

Durant l'année académique 2018-2019, nous avons traduit la première moitié de ce « Traité », dont il n'existe aucune traduction française. Dans la continuité des travaux commencés par J. Ware, Tsukamoto Zenryū et L. Hurvitz ${ }^{1}$, nous avons entamé une nouvelle analyse de cet ouvrage. Cela nous a permis de reparcourir les différentes étapes de l'histoire du bouddhisme en Chine du Nord, d'étudier son implication dans la politique et l'économie, et de préciser la formation de sa tradition littéraire, ainsi que son évolution vers des formes spécifiques. Tout d'abord, la lecture de cette partie du «Traité » nous a permis d'identifier un éventail de différentes catégories d'informations recueilles dans ce texte. Celle-ci peuvent être regroupées de la manière suivante : 1. Informations sur l'introduction du bouddhisme en Chine; 2. Notions de

1. James R. Ware, « Wei Shou on Buddhism », T'oung Pao, 30, fasc. 1-2 (1933), p. 100-181; Tsukamoto Zenryū 塚本善隆, Shina bukkyōshi kenkyū, Hokugi hen, 支那佛教史研究, 北魏篇, Tōkyō, Kōbundō, 1942; Leon Hurvitz, «Wei Shou, Treatise on Buddhism and Taoism », dans S. Mizuno, T. Nagahiro, Yün-kang: The Buddhist Cave-temples of the Fifth Century A.D. in Northern China, Kyōto, Jimbun kagaku kenkyūsho, 1956. 
base de ce système de pensée; 3 . Informations à caractère pratique et théorique sur l'organisation hiérarchique et fonctionnelle de la communauté monastique, sur les institutions liées à sa gestion, ainsi que sur la communauté des fidèles; 4. Informations concernant les modalités de diffusion de cette religion chez les Wei du Nord et implications sur l'histoire et la politique de la dynastie; 5. Aspects économiques et sociaux liés à la diffusion et à la popularisation de cette religion durant l'époque en question; 6. Informations sur la construction de temples, monastères, complexes rupestres et autres infrastructures liées au culte bouddhiste ou à l'organisation de la communauté religieuse. Nous avons essayé de déterminer les sources de ces données et d'identifier les citations tirées de textes spécifiques liés à la tradition scripturale bouddhiste. Une analyse croisée de ces sources nous a permis de clarifier certains passages complexes figurant dans ce texte, ainsi que de formuler des observations complémentaires sur les personnages et les évènements historiques mentionnés dans le « Traité ». Nous avons analysé plus en détail la section du texte qui fournit des informations sur les faits ayant amené à la persécution du bouddhisme (446-452) par l'empereur Taiwu 太武帝 (r. 423-452), sur la « renaissance » de la communauté religieuse et la construction d'importants sanctuaires rupestres dans la Chine du Nord durant les règnes des trois empereurs qui lui ont succédé, Wenchengdi 文成帝 (r. 452-465), Xianwendi 獻文帝 (r. 465-471) et Xiaowendi 孝文帝 (r. 471-499). Enfin, nous nous sommes concentrés sur les aspects sociaux et économiques liés au développement des pratiques et des institutions bouddhiques durant cette phase spécifique de l'histoire des Wei du Nord.

\section{Scènes de damnation sur les peintures murales de Dunhuang : inscriptions et éléments iconographiques}

La seconde partie de la conférence a été consacrée à une analyse préliminaire des représentations iconographiques portant sur l'imaginaire des mondes infernaux et des inscriptions associées à celles-ci sur les peintures murales de Mogao (Dunhuang). Cette étude nous a permis d'obtenir un aperçu global des sources disponibles et des typologies principales de représentations, à savoir les représentations des enfers à caractère " iconique ", et celles à caractère " descriptif » figurant dans des tableaux cosmologiques et dans des scènes illustrant différents sütra. Ce thème fera l'objet de nos conférences de l'année prochaine. Un regard croisé des sūtra canoniques et apocryphes comportant des descriptions des mondes de l'Au-delà nous permettra de mieux comprendre les éléments iconographiques figurant dans les représentations évoquées, et de justifier certains choix dans le programme ornemental des scènes de sūtra. 